\title{
Optical Camera Communication Performance Evaluation: Review
}

\author{
Noor J. Jihad ${ }^{1}$, Sinan M. Abdul Satar ${ }^{2}$ \\ ${ }^{I}$ Communication Engineering Department, University of Technology, Baghdad, Iraq \\ ${ }^{2}$ Electrical and Electronics Engineering department, University of Technology, Baghdad, Iraq \\ 30218@uotechnology.edu.iq,140006@uotechnology.edu.iq
}

\begin{abstract}
Recently, optical wireless communication (OWC) technologies focused on a camera or an image sensor receiver have drawn specific attention in areas like the internet, indoor localization, motion detection, and intelligent transportation systems. Besides, panorama sensors are the subject of communications from picture sensors as receptors as the high-speed OWC strategy do not need any change to the existing network, so the difficulty and expense of deployment are very limited. So in this paper, a detailed review of the techniques of optical camera communication (OCC)has been presented. In addition to their function of localizing, tracking and recording motion. Through addressing several facets of OCC and their different implementations, this study varies from the latest literature on this topic. The first section of the current article is on standardization, Path classification, modulation, scripting, synchronization, and signal processing methods for OCC networks whereas the second section of the research discusses OCC-based localization, navigation, motion detection, and smart transport systems literature .Finally, OCC's problems and potential work directions have been addressed in the final section of the research.
\end{abstract}

Index Terms - OCC optical camera communication, OWC optical wireless communication, camera communication, control with image sensors, localization, orientation, detection of movements.

\section{INTRODUCTION}

Wireless communication based optical range can address difficulties of transmission in a few usage situated fields extending from the family unit and manufacturing plant mechanical autonomy to vehicular systems. The lifetime and vitality productivity of lightemitting diode can be directed to various sources of light at an exceptionally clock rate [1]. The optical camera communication systems transmitter can be driven, a showcase, or advanced signage. Interestingly, the beneficiary is the image sensor from a computerized camera, webcam, or cell phone camera . Expanding request has prompted an expanding number of shrewd gadgets with installed cameras. Furthermore, the improvement of correlative (CMOS) complementary metal-oxide-semiconductor advancements makes an alternate age of high-pixel-goals and fast implicit camera.

These make huge open doors for OCC advancement. Especially, OCC innovation acquires and utilizes VLC visible light communication by utilizing an installed camera without altering equipment. The casing pace of most business camera items is 30 edges for/ each second (fps). To put it another way, the institutionalization of OCC focuses on the speculation of communication for both rapid just as low-speed camera beneficiaries. Soon, 
there are few rapid cameras that can uphold many frequencies per second [2]. Camera outline rates are anticipated to increment sooner rather than later attributable to ongoing headways in an image sensor innovationr; in addition to the pixel goals.

OCC is a networking device that uses optical image sensors as infrared IR recipients or visible bands, also referred to as image sensor communications [2, 3]. Second, the OCC consumers are set up on millions of pixels and can relay data to handle vast quantities of users using these pixels. Finally, the image sensors of current cameras may normally handle three colors to enable color transmission.

OCC has drawn much attention IoT, Indoor Localization, Motion Capture in environments such as the Internet, and intelligent Transportation Systems (ITS) because of such expense, popularity, and knowledge carrying capabilities. OCC has its drawbacks, , which include, though not limited to poor data quality due to the low recipient sampling quality, out-of-focus e picture, unreliable frame size. OCC has certain benefits when opposed to other forms of OWC optical wireless communication technology.

\section{A. Comparison between visible light communication and optical camera communication}

In this section, the comparison between visible light communication and optical camera communication will be illustrated. This comparison will be in terms of transmission distance, Wavelength, SNR, Receiver, Decoding, Data rate, Protocol, and MIMO Multiple inputs/multiple output Implementation.

TABLE (1) THE COMPARISON BETWEEN VLC AND OCC

\begin{tabular}{cr}
\hline Optical camera communication & Visible light communication \\
\hline Transmission distance of the OCC is up to several kilometers & Transmission distance of the VLC is Low \\
The wavelength used UV, IR, and Visible light. & The wavelength used is Visible light \\
Signal to noise ratio for OCC is high & Signal to noise ratio for VLC is low \\
The receiver type for OCC is the camera & The receiver type for VLC is the photodetector \\
The complexity of decoding is high & The complexity of decoding is low \\
The data rate is lower than VLC & The data rate is 11.67 kbps -96 Mbps \\
IEEE.802.15.7r1 protocol has been used for OCC & IEEE.802.15.7 has been used \\
Easier MIMO Implementation & MIMO implementation is difficult.
\end{tabular}

It is noticeable that OCC has many advantages compared with VLC so nowadays the studies trend has been directed toward the OCC in OWC systems.

\section{B. Area of focus}

The expanding requests for optical camera communication usage bring the distributions of a couple of summarized reviews on coding and modulation techniques for OCC. The vast majority of the current overview of OCC has been concentrating on coding and modulation schemes.

As proposed in [3], the balance system, preferences, and constraints of OCC are presented. Moreover, the article is centered on tending to the shortcomings of OCC, for example, camera inspecting rates, outline rate varieties and movement adjustment. Likewise, in [4], Saha et al introduced a study concentrating on the fundamental innovation thought in IEEE.802.15.7r1 assignment bunch for OCC frameworks. In [5], the creators have displayed a review on the uses of OCC in insightful transportation systems ((ITS), V2V vehicle to vehicle communications, vehicular to a system (V2I) interchanges 
coherent optical wireless communication area Communications (IOV), and so on.

A similar investigation has been exhibited in [5] which likewise incorporates potential designs and applications of OCC. Furthermore, review works committed to OCC were introduced $[6,7,8]$ explored the OCC proposition of IEEE $802.15 .7 \mathrm{~m}$ task gathering, indicated the basic specialized thought of the OCC specifications and talked about the next study trends. A comparable methodology was presented in [7] where the author has introduced the structure of OCC. Moreover, the author in [8], checked on various coding techniques.Also, talked about the specific edge structures for each modulation system. The examination between the interests of the studies referenced above is displayed in Table 2. In this study, the current writing on OCC was spread, including institutionalization, channel characterizations, synchronization, modulation, coding, restriction, route, movement catch, and insightful transportation systems. It has been agreed upon the factt that every one of these parts of OCC is included into a review structure and it makes the current research a remarkable commitment to the examination networks taking a shot at OCC, confinement, and movement catch based insightful transportation systems.

TABLE (2) COMPARISON OF THIS PAPER WITH PREVIOUS RESEARCHES

\begin{tabular}{ccc}
\hline Research area & Year & Reference \\
\hline Structure and implementation & 2015 & Saha et al [4] \\
Recipient models & 2017 & Bae et al. [3] \\
slam et al. [5] \\
OCC for the internet of a vehicle (IOV) & 2017 & Le et al. [6] \\
Structure and implementation & 2017 & Chowdhury et al. [5] \\
Models and applications & 2018 & Nguyen et al. [7] \\
Coding and modulation techniques & 2018 & Luo et al. [7] \\
Coding and modulation techniques & 2018 & Nasir et al.[8 ] \\
localization & & This paper \\
Standardization ,modulation, synchronization, & 2019 & \\
, coding, navigation, channel characterization & 2020 & \\
Design, implementation and future research directions & & \\
\hline
\end{tabular}

\section{DIFFERENT MODULATION TECHNIQUES FOR OCC}

In this part, various modulation techniques have been displayed. Moreover, various modulation techniques were featured for OCC. To give more explanation to the direction of enhancing the transmission distance and the data rate are not institutionalized at this point [9] by utilizing MIMO frameworks to improve the collector plan. Furthermore, an image sensing with the MIMO system was utilized in [10] to decrease the channel obstruction between the RGB signals. Moreover, a half and half balance plan of frequency shift keying and phase shift keying were researched in [11] where $\mathrm{M}$ is for different client access, and $\mathrm{N}$ is for expanding the information rate. To adapt the foundation encompassing, the author in [12] offered a crossbreed adjustment plan of spread range.

The suggested models in [13] offered optical camera communication by utilizing the watermarks implanted in the pictures. The offered thought in [14] has been tried by utilizing an LCD as a webcam as a beneficiary. A comparative optical camera communication was created in [15] which is hearty to camera turn, show size, impediment, mutilation, and obscuring the reachable information pace of discos was up to $1 \mathrm{kbps}$. As of late the proposed model in [16] built up an optical camera communication system called HYCACO which depends on spatial multiplexing and adventures the non-viewable pathway optical sign to enhance the information rate to $4.5 \mathrm{kbps}$. A LEDA2C was offered in [17] where close LED was utilized for transmission. 
The suggested plan in [18] was tentatively tried that accomplished centimeter-level restriction exactness and information pace of $6.72 \mathrm{kbps}$ for $100 \mathrm{~cm}$ transmission separation. The author in [19] suggested a quadric-chromatic LED-based OCC improve both light and information rate. The shading proportion system was utilized in [20] which had the option to accomplish the information pace of $13.2 \mathrm{kbps}$ a way off of $2 \mathrm{~cm}$. To enhance the data rate and separation of S2C interchanges, the author in [21] utilized a double camera collector with a split-screen technique. This strategy had the option to accomplish the information pace of $11.52 \mathrm{kbps} 2 \mathrm{~m}$. The data conveying capacities of OCC is additionally profoundly reliant on the sort of transmitters and recipients[22]. Because of the camera collector, include an altered OOK camera the feasible information rate for OCC systems is various where a huge assortment of exhibitions and trial has been led. The trial aftereffects of the writing and thought have been gathered in Table 3.

TABLE (3) OCC SYSTEMS PERFORMANCE COMPARISON

\begin{tabular}{|c|c|c|c|c|c|c|c|c|c|}
\hline $\begin{array}{c}\text { Modulation } \\
\text { schemes }\end{array}$ & $\begin{array}{l}\text { Data } \\
\text { rate }\end{array}$ & $\begin{array}{c}\text { Transmission } \\
\text { range (m) }\end{array}$ & sensor type & $\begin{array}{l}\text { Flick- } \\
\text { ering }\end{array}$ & $\begin{array}{c}\text { System } \\
\text { design }\end{array}$ & Synchronization & $\begin{array}{c}\text { Bits per } \\
\text { frame }\end{array}$ & Complexity & Ref. \\
\hline UPSOOK & $\begin{array}{l}0.15 \\
\text { kbps }\end{array}$ & 12 & Global shutter & $\mathrm{N}$ & LED2C & $\begin{array}{c}2 \text { bits } \\
\text { synchronization }\end{array}$ & $1 / 2$ & Low & 9 \\
\hline OOK & 2 bps & 25 & Rolling shutter & $\mathrm{N}$ & LED2C & $\begin{array}{c}2 \text { bits } \\
\text { synchronization }\end{array}$ & Multi & Low & 10 \\
\hline $\begin{array}{c}\text { Spatially- } \\
\text { Modulated } \\
\text { Space- } \\
\text { Time (SM-ST) }\end{array}$ & $1 \mathrm{kbps}$ & 30 & Rolling shutter & $\mathrm{N}$ & LED2C & $\begin{array}{c}\text { Mapping } \\
\text { synchronization }\end{array}$ & Multi & high & 11 \\
\hline UPAMSM & $0.1 \mathrm{kbps}$ & 50 & Global shutter & $\mathrm{N}$ & LED2C & $\begin{array}{l}\text { Mapping and } \\
\text { synchronization }\end{array}$ & $1 / 2$ & Moderate & 12 \\
\hline $\begin{array}{l}\text { Layered Space- } \\
\text { Time Code } \\
\text { (L-STC) }\end{array}$ & $1 \mathrm{kbps}$ & $40-210$ & Rolling shutter & $\mathrm{Y}$ & LED2C & $\begin{array}{c}\text { IEEE } 802.3 \text { start } \\
\text { bits }\end{array}$ & $1 / 2$ & high & 13 \\
\hline Color Barcodes & $\begin{array}{c}0.12- \\
0.96 \\
\text { Mbps }\end{array}$ & $0.12-0.24$ & Global shutter & $\mathrm{Y}$ & LEDA2C & FDMA & NA & high & 14 \\
\hline CIM & $\begin{array}{l}112.5 \\
\text { kbps }\end{array}$ & 0.2 & Rolling shutter & $\mathrm{N}$ & $\mathrm{S} 2 \mathrm{C}$ & $\mathrm{n} / \mathrm{a}$ & Multi & high & 15 \\
\hline $\begin{array}{c}\text { CIM and } \\
\text { Rateless codes }\end{array}$ & $\begin{array}{l}317.3 \\
\text { kbps }\end{array}$ & 0.2 & Rolling shutter & $\mathrm{N}$ & $\mathrm{S} 2 \mathrm{C}$ & $\mathrm{n} / \mathrm{a}$ & Multi & Moderate & 16 \\
\hline CIM & $\begin{array}{r}12.8 \\
\text { kbps }\end{array}$ & 0.5 & Rolling shutter & $\mathrm{N}$ & $\mathrm{S} 2 \mathrm{C}$ & QR Code protocol & NA & high & 17 \\
\hline $\begin{array}{c}\text { Spatial- } \\
\text { Temporal } \\
\text { Comple- } \\
\text { mentary Frames } \\
\text { (S-TCF) }\end{array}$ & $\begin{array}{c}240 \\
\mathrm{kbps}\end{array}$ & 0.6 & Global shutter & $\mathrm{Y}$ & $\mathrm{S} 2 \mathrm{C}$ & $\mathrm{n} / \mathrm{a}$ & NA & high & 18 \\
\hline $\begin{array}{c}\text { Pixel } \\
\text { translucency } \\
\text { modula- } \\
\text { tion }\end{array}$ & $\begin{array}{c}0.8-1.1 \\
\text { kbps }\end{array}$ & $0.3-1.5$ & Rolling shutter & $\mathrm{N}$ & $\mathrm{S} 2 \mathrm{C}$ & $\mathrm{n} / \mathrm{a}$ & NA & high & 19 \\
\hline CIM & $\begin{array}{l}11.52 \\
\text { kbps }\end{array}$ & 2 & Rolling shutter & $\mathrm{N}$ & $\mathrm{S} 2 \mathrm{C}$ & $\begin{array}{l}\text { Simple threshold } \\
\text { method }\end{array}$ & $1 / 2$ & high & 20 \\
\hline $\begin{array}{l}\text { Spatial Discrete } \\
\text { Multitone } \\
\text { (SDMT) }\end{array}$ & $\begin{array}{l}1.344 \\
\text { Mbps }\end{array}$ & 2 & Global shutter & $\mathrm{N}$ & $\mathrm{S} 2 \mathrm{C}$ & $\mathrm{n} / \mathrm{a}$ & Multi & high & 21 \\
\hline OFDM & $\begin{array}{c}12 \\
\text { Mbps }\end{array}$ & 10 & Rolling shutter & $\mathrm{N}$ & $\mathrm{S} 2 \mathrm{C}$ & $\mathrm{n} / \mathrm{a}$ & Multi & high & 22 \\
\hline
\end{tabular}




\section{SIMULATION RESULTS WITH MATLAB}

The schematic system diagram is shown in Fig.1. Light-emitting diode LED square measure mounted on the ceiling informs downwards to the ground level. A camera is employed to capture the image of the LEDs. To verify the mentioned technique, a camera simulation has been used that offers North American country the image of the LEDs in an exceedingly specific position. So, the pc vision system tool chest has been used in MATLAB. It additionally called the principal purpose, and therefore the skew coefficient and the unessential parameters: comprise a rotation $\mathrm{R}$ and a translation $\mathrm{t}$.

The origin of the camera's coordinates system is at its optical center and its X-and coordinate axis outline the image plane (MATLAB help). The standardization algorithmic rule calculates the camera matrix mistreatment of unessential and intrinsic parameters. Once the camera matrix has been gotten. This property at the side of the obtainable MATLAB codes square measure accustomed simulate a camera and therefore the technique made public higher than. As an example, Fig. 1, shows a ceiling with mounted LEDs (i.e., the $\mathrm{Z}$ coordinate is fastened on the coordinate of a thousand $\mathrm{mm}$ ). Fig. 8, shows the situation of the camera at the coordinates of $(500,500$, zero $\mathrm{mm})$ within the universe.

Basically, the camera orientation angle will be amenment and there is no care if the camera is the line of sight with LEDs or not, during this example, there is a tend to think that the camera is in line of sight with LEDs and horizontally flat. The simulation results of the mentioned camera provides the image of LEDs shown in Fig. 1. Now, there is coordinates of LEDs in pixels. Finally, AN optimization downside has been made and solved by the Imperialist Competitive algorithmic rule and if the parameters of the camera square measure chosen ideally, the calculable position, can precisely the same of the position of the camera within the universe and it shows that the current projected algorithmic rule works.

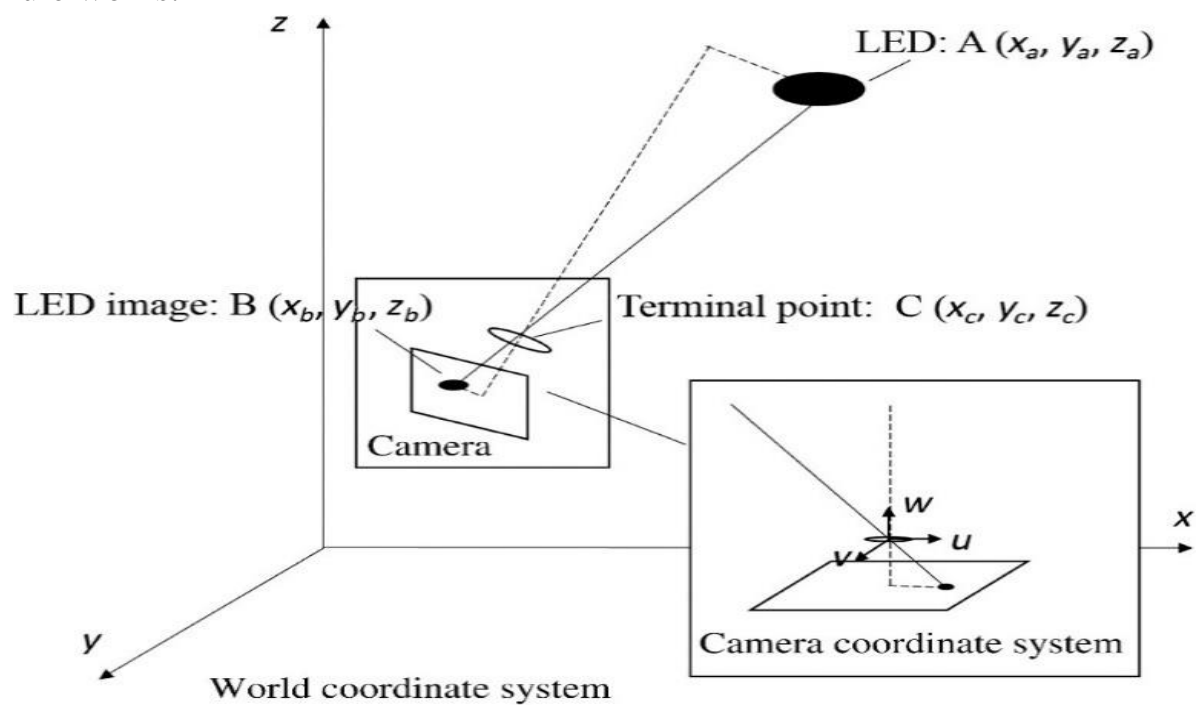

Fig. 1. THE SYSTEM BLOCK DIAGRAM LED COORDINATES IN THE REAL WORLD ERROR! REFERENCE SOURCE NOT FOUND..

Here are some results obtained from this simulation. First, the ideal camera is considered and the position of the camera in 18 points is changed. Fig. 2 shows the result of the simulation.

Generally, TXs need to be seen in a particular position to substantiate the proposed method. Once the parameters of the matrix of the camera are obtained, the 3-d environment scenario can then be projected to the picture plane as in [23]. In this work, a camera with 
the parameters given in Table 4 is simulated using the MATLAB. In other words, with MATLAB a camera was modeled by using the main parameters in the row. Moreover, the point of view of the photographs of LEDs is determined in keeping with the LEDs mounted at the room ceiling and the LEDs captured at a specific location on the level floor. Remember that the approach suggested here refers to the question of a specific goal optimization.

TABLE (4) SYSTEM PAPMETERS

\begin{tabular}{cc}
\hline Parameter & Value \\
\hline Room size & $2 \times 2 \times 2 \mathrm{~m}^{3}$ \\
No. of LEDs & Variable from 3 to 6 \\
Image sensor resolution & $1000 \times 1200($ pixel $)$ \\
Image sensor size & $22.4 \times 15(\mathrm{~mm})$ \\
Pixel size & $0.0224 \times 0.0125(\mathrm{~mm})$ \\
Focal length & $20(\mathrm{~mm})$ \\
Principal points & $500 \times 600($ pixel $)$ \\
\hline
\end{tabular}

Figure. 2 Contrasts the precise and approximate positions of the individual at 16 separate places with six LEDs mounted at a height of $2 \mathrm{~m}$ above the floor in the middle of the ceiling. As can be seen, there is a rather close correlation between the actual and approximate locations, with almost no deviations.

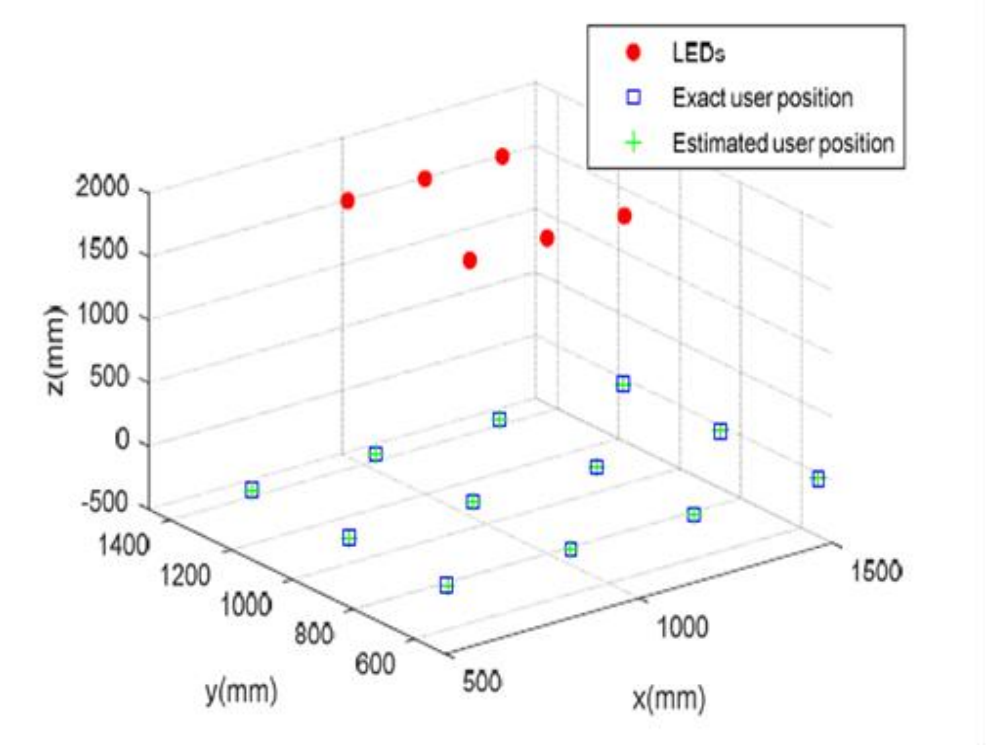

FIG. 2. THE DEVICE LOCATIONS ARE APPROXIMATE AND PRECISE.

It is worth remembering that in this situation all six LEDs are caught by the camera in the pictures taken at. Regarding the location; although our approach demonstrates a strong PE with an ideal virtual camera, it is important to understand both the radial and tangential lens distortions [19] utilizing actual cameras. The first is when the light rays move more similar to the sides of a lens than their center, while the latter happens because the lens and the image sensor are not parallel. Finally, it shows the distribution of PE inside the room in Fig.3. In the middle of the space, there is almost no PE but PE rises dramatically at the 
corners; this is attributed to the camera taking pictures with the decreased amount with LEDs.

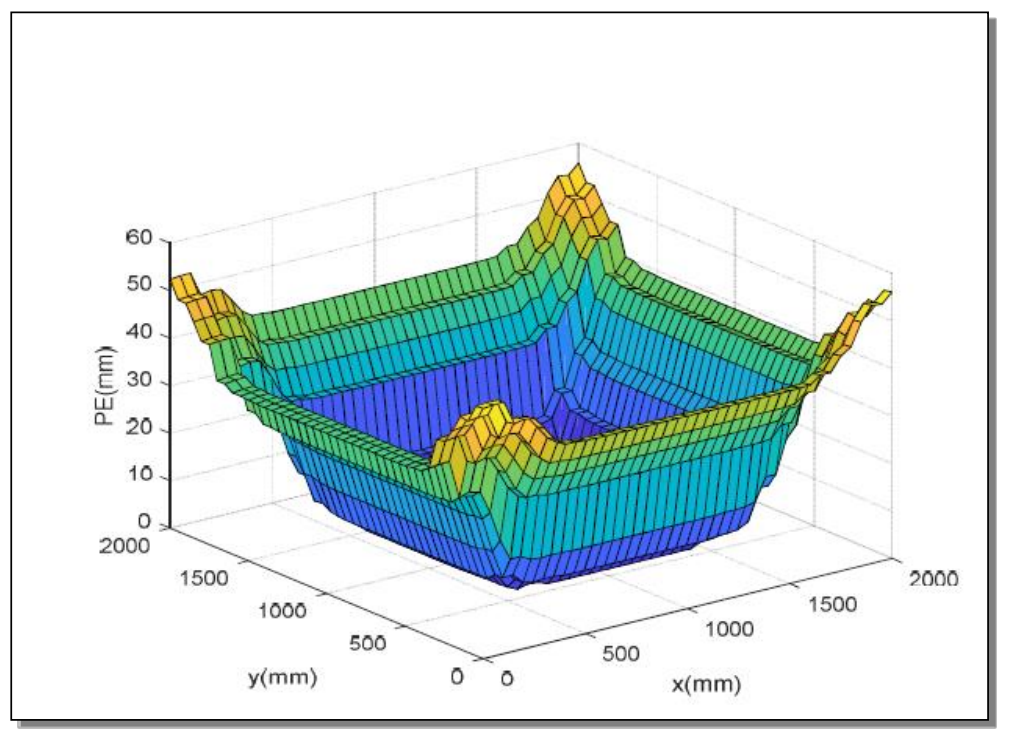

FIG. 3. DisTRIBUTION OF PE WITHIN THE ROOM.

Tangential distortion happens once the lens and therefore the image plane doesn't seem to be parallel. Each of them creates a displacement of points in a picture. On the opposite hand, lens distortion makes a displacement in pixels. So, if there is a tend to apply a touch lens distortion, the error in positioning will be seen. Fig. 4 shows the results of the positioning formula within the presence of the lens distortion. For the newest case, Fig.4, shows the error in x, y, and z-direction by applying lens distortion.

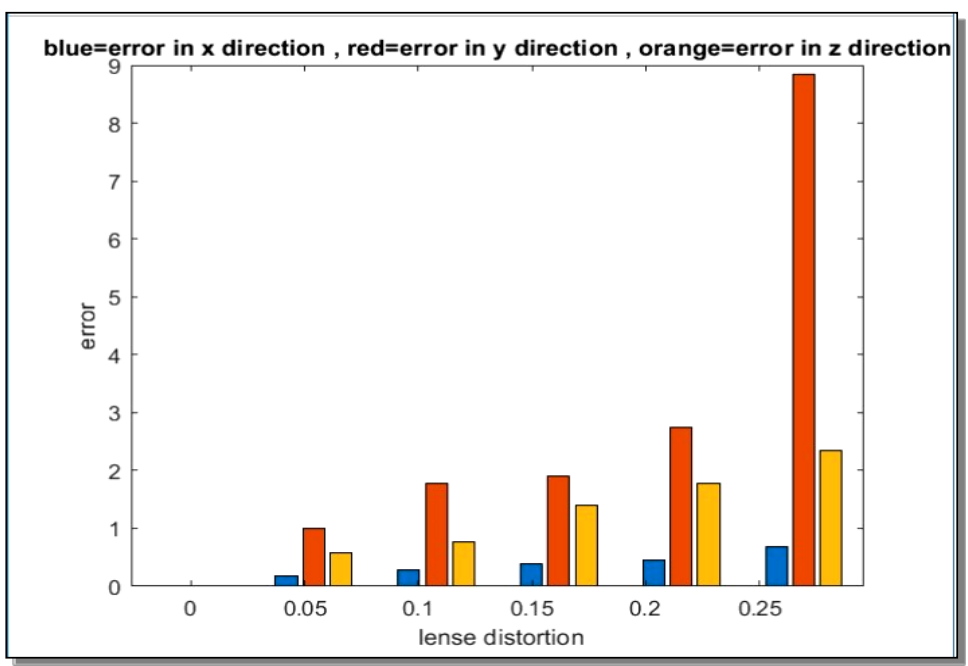

FIG. 3. ERROR IN X, Y, AND Z DIRECTIONS.

\section{CONCLUSION}

As a thought about a contender for the correction of 802.15.7, OCC innovation includes a significant plan of action that utilizes the points of interest of the VLC system. This investigation gives a review of the optical camera communication system from design to regulation and application structure. This modern worldview was contrasted and modern trend, scholarly and mechanical investigations on OWC. A base-up survey of paper substance was mentioned to give a top to bottom diagram of the OCC investigate issues that 
are considered for institutionalization. Generally speaking, an examination was clarified utilizing different execution viewpoints. The investigation of the image sensor engineering and thought of upcoming patterns shows that OCC introduces another assistance innovation for future wireless communication.

\section{REFERENCES}

[1] B. Zhu, J. Cheng, Y. Wang, J. Yan, and J. Wang, "Three-Dimensional VLC Positioning Based on Angle Difference of Arrival With Arbitrary Tilting Angle of Receiver," IEEE Journal on Selected Areas in Communications, vol. 36, pp. 8-22, 2018.

[2] K. Pahlavan, X. Li, and J.-P. Makela, "Indoor geolocation science and technology," IEEE Communications Magazine, vol. 40, pp. 112-118,2002.

[3] B. Mungamuru and P. Aarabi, "Enhanced sound localization," IEEE Transactions on Systems, Man, and Cybernetics, Part B (Cybernetics), vol. 34, pp. 1526-1540, 2004.

[4] C. V. Lopes, A. Haghighat, A. Mandal, T. Givargis, and P. Baldi, "Localization of off-the-shelf mobile devices using audible sound: architectures, protocols, and performance assessment," ACM SIGMOBILE Mobile Computing and Communications Review, vol.10, pp. 38-50, 2006.

[5] B. Lin, Z. Ghassemlooy, C. Lin, X. Tang, Y. Li, and S. Zhang, "An indoor visible light positioning system based on optical camera communications," IEEE Photonics Technology Letters, vol. 29, pp.579-582, 2017.

[6] A. T. Arafa, "An indoor optical wireless location comparison between an angular receiver and an image receiver," University of British Columbia, 2015.

[7] Y. Hou, S. Xiao, M. Bi, Y. Xue, W. Pan, and W. Hu, "Single LED beacon-based 3-D indoor positioning using off-theshelf devices," IEEE Photonics Journal, vol. 8, pp. 1-11, 2016.

[8] N. B. Priyantha, A. Chakraborty, and H. Balakrishnan, "The cricket location-support system," in Proceedings of the 6th annual international conference on Mobile computing and networking, 2000, pp. 32-43.

[9] Y. Noh, H. Yamaguchi, and U. Lee, "Infrastructure-free collaborative indoor positioning scheme for time-critical team operations," IEEE Transactions on Systems, Man, and Cybernetics: Systems, 2016, pp.418-432.

[10] M. S. Rahman, M. M. Haque, and K.-D. Kim, "Indoor positioning by LED visible light communication and image sensors," International Journal of Electrical and Computer Engineering, vol. 1, pp. 161, 2011.

[11] N. U. Hassan, A. Naeem, M. A. Pasha, T. Jadoon, and C. Yuen, "Indoor positioning using visible led lights: A survey," ACM Computing Surveys (CSUR), vol. 48, pp. 20, 2015.

[12] G. Simon, G. Zachár, and G. Vakulya, "Lookup: Robust and Accurate Indoor Localization Using Visible Light Communication," IEEE Transactions on Instrumentation and Measurement, vol. 66, pp. 2337- 2348, 2017.

[13] J.-Y. Kim, S.-H. Yang, Y.-H. Son, and S.-K. Han, "High-resolution indoor positioning using light emitting diode visible light and camera image sensor," IET Optoelectronics, vol. 10, pp. 184-192, 2016.

[14] H. Huang, L. Feng, G. Ni, and A. Yang, "Indoor imaging visible light positioning with sampled sparse light source and mobile device," Chinese Optics Letters, vol. 14, pp. 090602, 2016.

[15] B. Lin, X. Tang, Y. Li, M. Zhang, C. Lin, Z. Ghassemlooy, et al.,"Experimental Demonstration of Optical Camera Communications Based Indoor Visible Light Positioning System," A, vol. 2, pp. 2.

[16] Y. Jiang, P. Tsai, Z. Hao, and L. Cao, "Automatic multilevel thresholding for image segmentation using stratified sampling and Tabu Search," Soft Computing, vol. 19, pp. 2605-2617, 2015.

[17] Y.-C. Cheng, J.-Y. Lin, C.-W. Yi, Y.-C. Tseng, L.-C. Kuo, Y.-J. Yeh, et al., "AR-based positioning for mobile devices," in Parallel Processing Workshops (ICPPW), 2011 40th International Conference on, 2011, pp. 63-70.

[18] M. Abdollahi, A. Isazadeh, and D. Abdollahi, "Imperialist competitive algorithm for solving systems of nonlinear equations," Computers \&Mathematics with Applications, vol. 65, pp. 1894-1908, 2013.

[19] W.-L. Du and X.-L. Tian, "An automatic image registration evaluation model on dense feature points by pinhole camera simulation," in Image Processing (ICIP), IEEE International Conference on, 2017, pp. 2259-2263. [20] S. Sheoran, P. Garg, P. K. Sharma, Location tracking for indoor VLC systems using intelligent photodiode receiver, IET Commun. 12 (13) (2018) 1589-1594.

[20] S. Sheoran, P. Garg, P. K. Sharma, Location tracking for indoor VLC systems using intelligent photodiode receiver, IET Commun. 12 (13) (2018) 1589-1594.

[21] J. Luo, L. Fan, H. Li, Indoor positioning systems based on visible light communication: State of the art, IEEE Commun. Surveys Tuts. 19 (4) (2017) 2871-2893.

[22] N. Saeed, H. Nam, T. Y. Al-Na_ouri, M.-S. Alouini, A state-of-the-art survey on multidimensional scaling based localization techniques, IEEE Communications Surveys Tutorials (2019) 1-1.

[23] Shashikant, P. Garg, P. K. Sharma, Interference mitigation technique with coverage improvement in indoor VLC system, Trans. on Emerging Telecommun. Technol. 30 (2) (2019) 1-12. 